niste c'est-à-dire pour le consommateur. Ils ne sont pas sans valeur non plus du point de vue commercial, puisque le lait que l'analyse microbiologique dénonce comme pauvre en microbes est un produit capable de se conserver dans de bonnes conditions.

L'analyse microbiologique n'est pas un complément de l'analyse chimique, c'est un moyen d'information nouveau, au moins aussi nécessaire que l'autre et sans lequel le contrôle du lait ne s'exerce que sur une face. L'analyse chimique eontraint le vendeur du lait à l'honnêteté, l'analyse microbiologique oblige le laitier à entretenir des animaux en bonne santé, à assurer la propreté de son établissement et de son matériel, à être approvisionné en eau non contaminée. L'analyse microbiologique tient à l'hygiène du lait et de sa production ; on ne saurait nier qu'elle constitue un facteur puissant d'amélioration des conditions de l'approvisionnement en bon lait dont chacun souhaite la réalisation.

Les « constantes 》 du lait ne doivent plus être considérées comme des indices suffisants de sa qualité; il est de toute nécessité de pouvoir inscrire en face le résultat des épreuves microbiologiques; c'est la condition indispensable d'un contrôle qui doit viser tout autant à assurer la salubrité du lait que sa qualité marchande.

\title{
LA LUTTE GONTRE LA DISETTE DU LAIT PENDANT LA GUERRE 1914-1918 (suite et fin),
}

$$
\text { par le Dr A.-J.-J. VANDEVELDE, }
$$

Professeur de chimie générale à l'Institut Agronomique Supérieur de l'Etat, Directeur du Laboratoire chimique et bactériologique de la Ville de Gand, Directeur de l'Institut Supérieur des Industries de fermentation.

\section{III. - Répartition du lait et emploi des conserves.}

Dans le but de pouvoir parquer le lait naturel et de pouvoir le conduire à sa destination véritable, le nourrisson, il fallait attirer l'attention du public sur l'utilisation des poudres de lait et conserves de lait sous forme de lait évaporé et de lait condensé sucré que le Comité National de Secours et d'alimentation mettait à la disposition de la population. Dans un tract répandu en grande quantité, la Commission scientifique instruisit le public sur la valeur alimentaire de ces produits, en même temps que celle du lait centrifugé et du lait battu, en recommandant de n'employer le lait complet que pour les nourrissons, et de resireindre la fabrication du beurre: dans une 
période de disette, l'enfant doit rester le dernier consommateur du lait entiar de vache. Les poudres de lait, si elles ne peuvent facilement reconstituer du lait d'apparence normale quand on les mêle avec de l'eau, sont parfaitement utilisables en mélange avec du lait centrifugé, avec du lait battu, avec des bouillons et des soupes de légumes; elles peuvent servir à la préparation de panades très nutritives et très digestives, entrer dans la confection du pain, et, d'une manière générale, de tous les aliments ; elles peuvent même être consommées à l'état de poudre.

En raison de la disette, il était regrettable de ne pouvoir utiliser les próduits qui, en été, notamment, coagulaient par la chaleur par acidification. L'emploi de carbonate de sodium permettait souvent de remédier à cet inconvénient, et nous fûmes obligés d'admettre que, dans certains cas, on ajouterait une quantité de carbonate de sodium telle qu'il se produise au maximum $1 \mathrm{gr}$. de lactate de calcium par litre. Cette mesure n'était pas légale, mais que faire quand l'occupant accaparait tout, au point que la Commission scientifique fut obligẹe, le 10 décembre 1917, de protester énergiquement, mais sans résultat, en dénonęant que, pour les enfants jusqu'à 6 ans, la quantité de lait disponible était insuffisante, que les malades ne recevaient qu'un demi-litre de lait centrifugé par jour, et que le ravitaillement des habitants au-dessus de 6 ans, vieillards compris, ne pouvait repartir entre 200.000 personnes que 736, , litres de lait centrifugé, soit $3,5 \mathrm{~cm} .3$ (trois centimètres eubes et demi) par personne et par jour !

Les gouttes de lait administrées par des dames dévouées rendirent les plus grands services en assurant la répartition du lait pour les enfants; l'hygiène infantile, gràce aux conseils eonstants donnés aux mères, s'en ressentit beaucoup; la propreté des enfants et des récipients apportés pour le transport du lait furent surveillés de très près.

Quoique tout désinfectant fut sévèrement proserit, nous fùmes, à un certain moment, contraints par les circonstances à faire un essai de nettoyage de bouteilles de distribution par le formol. L'essai fut fait sous contrôle médical et chimique. Les bouteilles nettoyées suivant les méthodes ordinaires étaient rincées une dernière fois avee une solution faible de formol à 1 p. mille, puis renversées sur des elaies pour les sécher en vue de la distribution du lendemain. Le contrôle chimique ne permit pas de retrouver des traces de formol dans le lait, et la surveillance clinique des nourrissons ne permit d'observer aucun phénomène spécial. Le procédé demande nécessairement une surveillance constante ; il fut suivi environ pendant deux mois dans l'un des locaux de distribution de la goutte de lait. II permit, en plein été, une conservation plus longue du lait pasteurisé distribué par les bidons du Service de haute pasteurisation. 
Si nous devions, dans le but de veiller au ravitaillement en lait des enfants, parfois combattre l'excès de l'acidité du lait par l'emploi de carbonate de sodium - et dans ee cas, les quantités de réactif étaient soigneusement dosées, - nous devions veiller aussi à ce que le lait fourni présente à ce sujet le degré de pureté le plus complet. Je me suis donc préoccupé d'établir avec certitude l'addition frauduleuse de carbonate de sodium.

Dans une note (1) publiée récemment, j'ai fait connaître en même temps que l'état de la question à laquelle M. Porcher (2) notamment a consacré un intéressant travail, un procédé de détermination de l'addition de carbonate de sodium au lait; ce procédé est basé sur le rapport entre la teneur en métaux alcalins et la teneur en anhydride phosphorique dans les cendres du lait ; ces cendres contiennent, en effet, en moyenne autant de sodium et de potassium $(\mathrm{Na}+\mathrm{K}=6$ $+20,4=26,4 \%)$ que d'anhydride phosphorique $\left(\mathrm{P}_{2} \mathrm{O}_{3}=26,3 \%\right)$.

L'introduction de carbonate, ou de bicarbonate de sodium, a pour effet d'augmenter la teneur en métaux alcalins, et de modifier ainsi le rapport $\mathrm{Na}+\mathrm{K}=\mathrm{P}_{2} \mathrm{O}_{5}$. Le dosage se fait comme suit : Les cendres de $250 \mathrm{~cm}^{3}$ de lait sont dissoutes par un peu d'acide nitrique ; la moitié du liquide obtenu sert au dosage de Na et K, l'autre moitié au dosage de $\mathrm{P}_{2} \mathrm{O}_{5}$. Dans le dosage des métaux alealins, la solution est précipitée par un excès d'eau de baryte pour éliminer les phosphates, puis par du carbonate d'ammonium pour éliminer l'excès du baryum, ainsi que le calcium et le magnésium. Le filtrat est évaporé à sec, le résidu est repris par un peu d'eau, la solution traitée par un peu d'oxalate d'ammonium, fittrée, évaporée, incinérée légèrement, puis plus fortement après addition d'un peu d'acide sulfurique. Le dosage de l'anhydride phosphorique se fait selon les méthodes courantes.

Des essais effectués sur dés échantillons de lait additionnés de carbonate de sodium ont donné les résultats suivants :

\begin{tabular}{ccccc} 
Acidité N/10 & Essai à l'alcool & \multicolumn{3}{c}{ Métaux alcalins calculés en } \\
\cline { 2 - 4 } & $70 \%$ & $\mathrm{Na}$ & $\mathrm{K}$ & $\mathrm{P}_{2} \mathrm{O}_{6}$ \\
- & - & - & - & - \\
$11,5 \mathrm{~cm}^{3}$ & Coagulation & $23,8 \%$ & $33,0 \%$ & $15,8 \%$ \\
$10,0 \mathrm{~cm}^{3}$ & id. & $39,2 \%$ & $56,1 \%$ & $10,8 \%$
\end{tabular}

Grâce à ce procédé, je suis parvenu souvent à établir avec certitude l'addition de carbonate de sodium, addition que très souvent la coagulation dans l'essai à l'alcool à $70 \%$, se produisant en même temps qu'une faible acidité, permettait de supposer.

(1) A.-J.-J. VANDEVELDE. - La recherche de l'addition de carbonate de sodium au lait. Bull. Soc. Chim. Bel., 1920, 29, p. 52.

(2) C. Poncher et A. Bonis. - Le dosage du lactose dans les laits chauffés après addition de carbonate de sodium. Ann. Falsif., 1919, 11, pp. 195 et 295. 
Je désire, pour terminer ce travail, relater le résultat d'un essai que j'ai pu effectuer en vue d'établir si un étamage plombifère des appareils de chauffaga et de transport du lait peut introduire dans le lait des quantités appréciables de plomb (1). Cet essai avait de l'importance pendant la guerre parce que l'étain pur était rare, souvent introuvable, et qu'il était nécessaire, en cas d'utilité, de pouvoir établir jusqu'à quel point l'étain plombifère pouvait être utilisé.

Dans l'expérience que je vais décrire, les conditions de conservation ont été exagérées dans le sens défavorable; le bidon d'essai était intérieurement étamé à l'aide d'étain contenant $7,1 \%$ de plomb. Le bidon neuf duquel l'alliage avait été enlevé partiellement par raclage, présentait done une surface attaquée ; après avoir rempli d'eau et chauffé, ee bidon fut après un triple rinçage à la vapeur. rempli de 20 litres de lait écrémé, et soumis pendant 30 minutes à la haute pasteurisation. Sans aucun refroidissement, le bidon a été abandonné à lui-même pour permettre le développement éventuel des spores présentes ; le lendemain la pasteurisation fut renouvelée, mais cette fois suivie de refroidissement énergique réglementaire.

A l'ouverture du bidon, j'ai constaté, ainsi qu'il fallait s'y attendre, que le lait était coagulé, résultat du manque de refroidissement après la première pasteurisation. Ce lait est resté dans le bidon encore deux jours avant d'être soumis à l'analyse. Les conditions d'attaque du métal d'étamage sont done intentionnellement exagérées; les conditions normales sont : 1 à 2 jours de séjour, refroidissement après le chauffage, lait normal, non acide.

La recherche du plomb étant délicate et devant conduire à un dosage difficile, je n'ai pas hésité à mettre en œuvre la masse totale du lait travaillé, soit 20 litres, quoique cette manipulation dans du matériel chimique soit plutôt désagréable. Mais l'emploi d'une forte quantité de substance permettait de doser plus facilement le plomb dans le cas ou il aurait été présent.

La première opération consiste naturellement dans la destruction de la matière organique. Cette opération peut se faire notamment par évaporation et incinération, ou par traitement par l'aeide chlorhydrique et le chlorate de potassium, ou par l'acide nitrique concentré.

J'ai rejeté de suite le procédé par incinération, cependant le plus rapide et le moins onéreux (point important au cours d'une disette de réactifs), parce qu'il peut permettre lélimination du plomb sous forme de chlorure de plomb assez volatil ; j’ai rejeté aussi le traitement au chlorate qui augmente la quantité des matières minérales

(1) A.-J.-J. VANDEVELDE, - L'atlaque de l'étain et de l'étain plombifère par le lait. Bull. Soc. Med., Gand, 1919, pp. 142-152. 
que j'avais intérêt à éviter le plus possible : il ne faut pas perdre de vue que 20 litres de lait doivent produire $140 \mathrm{gr}$. de cendres, dont la moitié est constituée de phosphates.

J'ai donné la préférence à l'acide nitrique qui produit une oxydation très régulière, quoique lente, d'autant plus que j'ai décrit autrefois un mode de dosage de l'étain dans certaines denrées alimentaires (1), qui m'a donné toute satisfaction, et qui est basé sur l'emploi de ce réactif. J'ai dủ me résigner à sacrifier à cette recherche $8 \mathrm{kgr}$. d'acide nitrique pur et concentré,ce qui dans le moment où ce produit est rare et conteux ne se justifie que par l'importance du renseigne. ment à obtenir.

En prenant les précautions d'usage, j'ai donc traité la masse totale par l'acide nitrique jusqu'à obtenir finalement après 15 jours de travail, environ un litre d'une masse épaisse à laquelle j'ai ajoulé $50 \mathrm{gr}$. d'acide sulfurique pur pour transformer le plomb en sulfate.

Il ne fallait pas songer à séparer par filtration la partie iusoluble, constituce, comme je l'ai reconnu, de sulfate de calcium et de combinaisons organiques nitrées, à cause de la concentration des acides, et j'ai préféré traiter toute la masse par l'ammoniaque. Je précipite, il est vrai, du même coup l'acide phosphorique qui se trouve présent en forte quantité, mais je ne perdais pas de vue, en même temps, que du plomb pouvait étre perdu sous forme de phosphate de plomb insoluble.

A la masse alcalinisée par l'ammoniaque, j'ai ajouté un excès d'acétate d'ammonium dans le but de dissoudre le sulfate de plomb, et-éventuellement un peu de phosphate de plomb, et j'ai soumis à la filtration. La partie insoluble a été épuisée ensuite à deux reprises par l'acétate d'ammonium et les liquides filtrés joints au premier filtrat. Le liquide total a été, après évaporation préalable, traité par le sulfure d'ammonium; le lendemain un précipité brun, noirâtre s'était formé et fut séparé par filtration. Après lavage, ce précipité a été dissous dans l'acide chlorhydrique concentré. Après évaporation de l'excès d'acide, reprise à chaud par l'eau distillée et filtration, j'ai soumis le liquide filtré à la précipitation par l'hydrogène sulfuré. Le sulfure obtenu a été ensuite transformé en sulfate, par traitement à l'acide sulfurique dans une capsule tarée ; j'ai obtenu ainsi $0,0098 \mathrm{gr}$. de sulfate de plomb contenant $0,0067 \mathrm{mgr}$. de plomb.

Le précipité du traitement alcalin pouvant contenir encore du plomb sous forme de phosphate, il était encore nécessaire de reprendre cette partie de substance par l'acide nitrique ; je mettais ainsi, il

(1) A.-J-.J. Vandevelde. - Dosage de l'étain dans le pain d'épices; Bull. Surv, denr. alim., 1904, 122-127. 
est vrai, de nouveau l'acide phosphorique en liberté, mais par contre, le plomb éventuellement présent, devenait nitrate de plomb. Après avoir donc traité prudemment par l'acide nitrique, la masse a été filtrée et le filtrat a été fortement dilué par des lavages répétés.

Après évaporation. pour chasser autant que possible l'acide nitrique, j'ai repris par l'eau et enfin traité le liquide par l'hydrogène sulfuré ? Je n'ai pu obtenir qu'un précipité jaune pâle de soufre, ne présentant aucun des caractères distinctifs du sulfure de plomb. Je dois en conclure que tout le plomb avait dû être extrait au cours du traitement à l'acétate d'ammonium.

J'ai donc pu extraire des 20 litres de lait mis en expérience, dans les conditions indiquées, $0,0067 \mathrm{gr}$ de plomb, ce qui représente $0,33 \mathrm{mgr}$. de plomb par litre. Cette quantité peut paraître faible, mais elle est cependant appréciable ; si elle ne s'élève qu'à $0,33 \mathrm{mgr}$. par jour, puisqu'une ration de lait pour enfant atteint au maximum un litre, elle représente par an $120 \mathrm{mgr}$. de plomb, et c'est une indication dont il faut tenir compte puisque le plomb s'élimine très lentement de l'organisme en s'accumulant dans certains organes, notamment le foie.

On devait done se poser, aux heures noires de l'occupation, cette question angoissante : l'étain pur faisant défaut pour l'étamage des eruches employées à la pasteurisation du lait, faut-il supprimer un service qui assure aux nourrissons la ration quotidienne, ou faut-il tolérer pour continuer d'assurer cette ration, l'emploi d'étain plombifère dans une proportion qui pourrait être limitée à $10 \mathrm{p} .100$ par exemple, et, dans ce cas, exposer les enfants à des intoxications ?

Devant ce grave dilemme, la Commission scientifique d'études pour la lutte contre la disette du lait, s'imposa de grands sacrifices pour obtenir. à l'insu de l'occupant, les quantités indispensables d'étain pur, à des prix exorbitants, il est vrai, mais en évitant ainsi d'outrepasser les tolérances de nos lois nationales pour motif de force majeure, et en évitant surtout de compromettre la santé des enfants, déjà si éprouvée par la guerre. 